
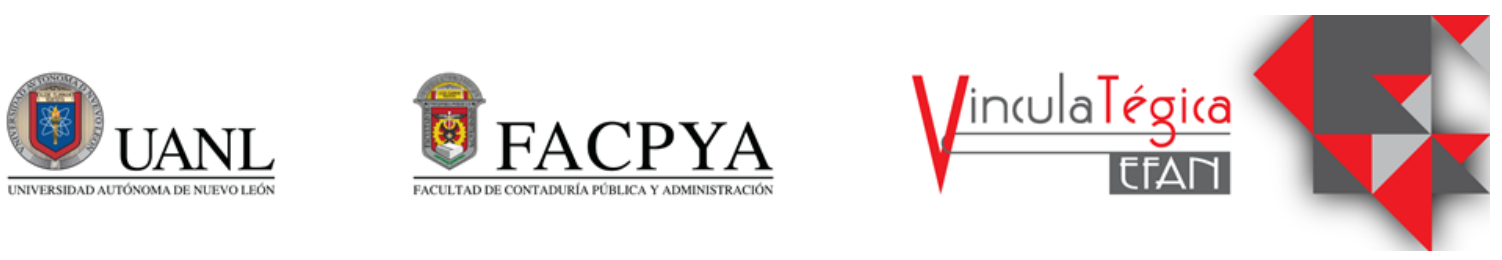

\title{
Panorama económico para el desarrollo del peso digital emitido por el Banco de México
}

\author{
Sergio Gabriel Ordóñez Sánchez ${ }^{1}$, César Alejandro García Franco ${ }^{2}$ y Axel Jesús Escoto \\ Flores $^{3}$
}

${ }^{1}$ Benemérita Universidad Autónoma de Puebla, Facultad de Contaduría Pública, Puebla, México, correo: sergio.ordonez@correo.buap.mx, Av.Universidad No.77 Col. Ciudad Universitaria, (+52) 2224558147

2Universidad Autónoma de Nayarit, correo: 18006260@uan.edu.mx, Ciudad de la Cultura "Amado Nervo", C.P. 63155, Tepic Nayarit, México. (+52)3112691167

${ }^{3}$ Benemérita Universidad Autónoma de Puebla, Facultad de Contaduría Pública, Puebla, México, correo: axel.escoto@alumno.buap.mx, Av. Universidad No. 77 Col. Ciudad Universitaria, (+52) 2282768126

Información del artículo revisado por pares

Fecha de aceptación: junio-2021

Fecha de publicación en línea: diciembre-2021

DOI: https://doi.org/10.29105/vtga7.1-130

\begin{abstract}
Resumen
Al paso de los años, la economía del mundo en el que vivimos va evolucionando a pasos agigantados, y a la par de este cambio en los ecosistemas, se ha podido apreciar la aparición de nuevas tecnologías derivadas del surgimiento de las criptomonedas, siendo la base para el desarrollo de las monedas digitales que serán emitidas por los bancos centrales de Europa y Asia. A través de encuestas aplicadas a personas inmersas en la utilización de los diferentes métodos de pago en la comunidad estudiantil, se realizó esta investigación con el objetivo de analizar el panorama de los métodos de transacciones electrónicos en México, para determinar el posible escenario que podría tener la creación del peso digital respaldado por Banxico, dando como resultado que existe una buena aceptación, sin embargo la sociedad mexicana no se encuentra preparada para hacerle frente a este tipo de cambio en la economía.

Palabras clave: Peso Digital, Panorama Económico, Monedas Digitales, Métodos de Pago
\end{abstract}

\begin{abstract}
Over the years, the economy of the world in which we live has evolved by leaps and bounds, and along with this change in ecosystems, it has been possible to appreciate the appearance of new technologies derived from the emergence of cryptocurrencies, which have served as basis for the development of digital currencies to be issued by central banks in Europe and Asia. Through surveys applied to people immersed in the use of different payment methods in the student community, the present investigation was carried out with the objective of analyzing the panorama of electronic transaction methods in Mexico, to determine the possible scenario that could have the creation of the digital weight backed by Banxico, resulting in a good acceptance, however, Mexican society is not prepared to face this type of change in the economy.
\end{abstract}

Keywords: Digital Weight, Economic Outlook, Digital Currencies, Payment Methods.

JEL: O33, O11, O42, E42 


\section{INTRODUCCIÓN}

Al paso de los años, la economía del mundo en el que vivimos va evolucionando a pasos agigantados, de tal manera que ha facilitado en muchos sentidos las transacciones a nivel global y a la par de este cambio en los ecosistemas, se ha podido apreciar la aparición de nuevas tecnologías derivadas del surgimiento de las criptomonedas, que son el punto de partida para la creación y desarrollo de las monedas digitales soberanas que buscan serán emitidas por los bancos centrales de Europa y Asia. Además, se perfilan para ser las principales monedas oficiales para la realización de transacciones.

A principios del 2021, China aumento gradualmente las pruebas y puesta en marcha de su primera moneda digital respaldada por el banco central, lo que la convertiría en la primer moneda digital soberana importante del mundo, la cual es conocida allí como DCEP (por "digital currency electronic payment" o pago electrónico en moneda digital). Por lo que, de esta forma, si el proyecto tiene éxito, se podrá eliminar la utilización y necesidad de efectivo físico y servicios de pago en línea como PayPal, sustituyendo las transacciones con un yuan digital.

Los bancos comerciales serán los encargados de distribuir el DCEP a sus clientes a través de billeteras digitales, y estos podrán retirar efectivo de forma como lo hacen al día de hoy en cajeros automáticos.

De esta forma, las personas podrán realizar pagos de servicios o adquirir productos sin la necesidad de mantener contacto con otros consumidores por medio de sus DCEP.

Así mismo, "emitir una moneda digital soberana podría ser una forma para que el gobierno recupere el control de las transacciones financieras nacionales", señala a DW, desde Beijing, Alexander Badenheim, gerente de proyectos de la fundación alemana Konrad Adenauer Stiftung (KAS) en la capital china. Por otra parte, el Banco Popular de China (PBOC) tiene la visión de utilizar este medio de pago disponible para los próximos Juegos Olímpicos de Inverno, programados para el mes de febrero de 2022 en Beijing.

Además, el millonario chino Chandler Gou, pionero de bitcoin, dijo a la $\mathrm{BBC}$ que, en el futuro, todo usarán DCEP (Forbes, 2020).

Así mismo, el Banco Central Europeo (BCE) estudia y analiza, de manera similar, la creación del Euro Digital basado en las fórmulas de la tecnología Blockchain, permitiéndole a Europa contar con herramientas entorno a un seguimiento transparente de la información, transacciones seguras y cualquier registro relacionado bajo el concepto de Central Bank Currency (CDBC en sus siglas en inglés).

Estaríamos en una "modalidad digital del dinero del banco central que es diferente de los saldos en la reserva tradicional o cuentas de liquidación y que depende directamente de la institución". Así mismo, desde el BCE se trabajan con medidas preliminares, actuando con cautela para que, a mediados de 2021, se obtengan los primeros estudios y pruebas relacionados a este proceso. (BBVA, 2021).

Por esta razón, es importante analizar el escenario que tendría una moneda digital que tuviera como respaldo el peso mexicano y que esta a su vez fuera emitida por el Banco de México, ya que, en un futuro no muy lejano, los especialistas en materia económica mencionan que hay una gran posibilidad de que paulatinamente se deje de utilizar el dinero físico, ya que cada una de las transacciones que se lleven a cabo se realizaran de manera digital, mediante la utilización de las monedas digitales.

\section{MARCO TEÓRICO}

2.1. Principales métodos de pago en México.

Existen dos principales sistemas de 
transferencias y pagos en México, el sistema SPEI y el sistema CoDI (Coutiño, 2020). En el año 2004 comenzó a operar en el país, el sistema de Pagos Electrónicos Interbancarios (SPEI) "es la infraestructura de pagos del Banco de México que permite a sus participantes enviar y recibir pagos entre sî para poder brindar a sus clientes finales el servicio de transferencia electrónico en tiempo real" (Banxico, 2018).

Además, es el sistema de transferencias más moderno y eficiente, el cual te permite ser utilizado desde cualquier dispositivo con conexión a internet.

Algunos años más tarde, en 2019, comenzó sus operaciones la nueva versión de transferencias electrónicas, conocida como CoDi, es una "plataforma de cobro digital que permite realizar pagos y cobros electrónicos en segundos, sin comisiones, sin necesidad de efectivo, de manera segura y eficiente desde la comodidad de un celular, las 24 horas del día sin interrupciones" (Santander, 2019).

Al cierre del 2019, se realizaron 5,321.7 millones de transacciones y transferencias según el informe presentado por la Comisión Nacional Bancaria y de Valores (CNBV, 2020). Sin embargo, se presentó una disminución en el importe de las transferencias interbancarias o al mismo banco, al pasar del $162 \%$ a $147 \%$ como porcentaje del PIB del cuarto trimestre de 2018, al mismo periodo de 2019.

Durante el periodo de octubre de 2019 a junio de 2020, las transferencias enviadas por CoDI se incrementaron considerablemente en número y monto (CNBV, 2020).

\subsection{Aceptación de las Fintech en} México.

El término "Fintech" deriva de las palabras "finance technology" y se utiliza para "denominar a las empresas que ofrecen productos y servicios financieros, haciendo uso de tecnologías de la información y comunicación, como páginas de internet, redes sociales y aplicaciones para celulares. De esta manera prometen que sus servicios sean menos costosos y más eficientes que los que ofrecen la banca tradicional" (CONDUSEF, 2017).

México ha sido considerado unos de los pioneros en América Latina en materia Fintech por la creciente cantidad de empresas que ha tenido durante los últimos años. Lo cual sería un detonante para agilizar los procesos de monitoreo y cumplimiento de las obligaciones tanto de las Fintech como de los participantes tradicionales de la industria y en efecto así ha sido al paso de los años (García Garza, Lanzagorta Sánchez, \& Ponce González, 2020).

Por lo que, para el 8 de marzo del 2018, entraría en vigor en México y América Latina la primera disposición oficial para regular este tipo de entidades, bajo la denominación de "Ley para Regular las Instituciones de Tecnología Financiera", que tendría el objeto de regular los servicios financieros que presten las instituciones de tecnología financiera, así como su organización, operación y funcionamiento (LEY FINTECH, 2018).

A pesar la creación de esta ley en materia regulatoria, no significo un impedimento el continuo aumento de las empresas Fintech, sino que al paso del tiempo el número total de empresas Fintech sigue creciendo en México, ya que, en promedio, el incremento fue de alrededor de $29 \%$ anual de 2016 a 2020.

Así mismo, de acuerdo con el Global Fintech Adoption Index 2019 de la firma EY, en México, el índice de adopción de servicios Fintech es del $72 \%$, lo cual representa que siete de cada 10 usuarios digitalmente activos utilizan opciones de tecnología financiera (Fintech) (Gutiérrez, El economista, 2019).

Lo que confirma, que al paso de los años la sociedad mexicana cada vez más acepta este tipo de servicios financieros proporcionados por las Fintech, sin embargo, "tienen el reto de seguir brindando confianza y seguridad a los usuarios para que los productos y servicios 
ofrecidos por empresas Fintech sean utilizados por los mexicanos de manera más frecuente, entendiendo que ya están reguladas por el gobierno para mostrar transparencia financiera, información clara y oportuna, sobre las obligaciones y derechos que poseen" (García Garza, Lanzagorta Sánchez, \& Ponce González, 2020).

\subsection{Inclusión financiera en México.}

En base a la Encuesta Nacional de Inclusión Financiera realizada en el 2018 por INEGI, arrojo que 54 millones de personas de 18 a 70 años (68\%) tienen al menos un producto financiero. Representan 2 millones más de personas con respecto a 2015.

Así mismo se incrementó la población con más de un producto financiero, donde las personas con 2 o 3 productos financieros aumentaron 2.6 millones. Y el $47 \%$ de los adultos en México tienen una cuenta en un banco o institución financiera (INEGI, 2018).

Por otra parte, tan solo el $22 \%$ de la población con cuenta formal de 18 a 70 años cuenta con banco por celular, mientras que el $78 \%$ que no tienen banco por celular, argumentan que el principal motivo por el que no contratan este tipo de servicios de banco por celular, es porque prefieren el uso de otros medios como son la sucursal, el cajero automático o el banco por internet, entre otros. Además, a la fecha la principal forma de pago de en el país para la realización de compras y pagos de servicios es el efectivo (INEGI, 2018).

Así mismo, en un estudio realizado por la CONDUCEF revela que de enero a junio del 2020 se realizaron alrededor de 1,634 millones de pagos con tarjetas en comercios tradicionales y en comercios electrónicos. Los pagos en comercios electrónicos representaron el $15.2 \%$ del total de pagos (CONDUSEF, 2020).

\subsection{Aceptación de las cirptomonedas en México. \\ Según el estudio "Criptómetro MX18: el primer estudio de criptomonedas en México",}

realizado en el 2018 por Psyma Latina, Wisum, Bitso y Fiinlab; reflejo que el 35\% de los mexicanos que ya cuenta con algún tipo de activo virtual, lo utiliza para realizar pagos o compras en línea, el 32\% decidió ahorrarlas en espera de que en algún momento aumente su valor y $15 \%$ prefiere utilizarlas como dinero digital para realizar pagos en establecimientos.

Dicho esto, México se colocó como uno de los países más avanzados en cuanto a criptomonedas se refiere en América Latina, siendo el tercero con más transacciones por detrás de Brasil y Colombia (Bitcoin México, 2020). A finales del 2019, un informe de la UENI revela que tan solo un $32.9 \%$ de las pequeñas y medianas empresas sabe qué son las criptomonedas, y de ese tercio escaso de pequeños negocios que las conocen, tan solo la mitad de ellos las aceptaría como medio de pago (Visión Industrial, 2020).

Así mismo, según la Encuesta Global de Consumidores de la firma alemana Statista, la cual se enfoca a recabar datos respecto a indicadores económicos y financieros, en México $12 \%$ de su población ha reconocido haber usado, o poseer, criptomonedas (Gutiérrez, El Economista, 2020).

Por otra parte, de la mano de la innovación tecnológica en economía mundial, y por causa del descontento ocasionado por la crisis económica de 2008, se crearon las criptomonedas, las cuales han marcado en gran manera el actual sistema financiero. Las cuales han permitido sentar las bases para los actuales proyectos en desarrollo de monedas digitales que serán emitidas por los bancos centrales de Asia y Europa. Así mismo, México no ha sido la excepción en cuanto a la aceptación de este tipo de activos virtuales, donde una parte de la población ya los conocen o han operado con alguna de ellas. Por lo que para un segmento de la población no sería algo nuevo, sin embargo, el desarrollo de una moneda digital en México que fuera emitida por Banco de México, marcaría un gran avance en el desarrollo de la economía. 


\section{MÉTODO}

Se realizó una investigación exploratoria realizando análisis, consultas y lectura de manera documental en diversos libros, artículos, revistas y paginas especializadas en temas de Inclusión Financiera, Métodos de Pago Digital, Fintech, Monedas Digitales.

La investigación se aborda con un enfoque de estudio de caso, utilizando un método cuantitativo y cualitativo, se apoya de un instrumento de estudio tipo encuesta de diseño propio, el cual se elaboró en apego a temas relacionados con el objetivo planteado, como los principales métodos de pago utilizados, la seguridad y confianza que brindan los métodos de pago digital, su margen de problemas en su utilización, así como los panoramas de seguridad que sentirían los usuarios al ya no poder utilizar dinero físico; realizando la aplicación de una prueba piloto previa, sometiendo los ítems a expertos en el área y realizando las modificaciones pertinentes para disminuir el riesgo de sesgo de información. el instrumento fue aplicado a una muestra de 170 personas que durante su vida cotidiana se encuentran inmersos en la utilización de los diferentes métodos de pago en México de una población de 1000 personas de diferentes comunidades estudiantiles, por lo que cuentan con la característica de usar constantemente los diferentes métodos de pago en el país, así como también tener el conocimiento sobre los constantes cambios tecnológicos en la economía, y además de que cada personas enfrenta un escenario diferente en cuento a la situación financiera que cada día les toca vivir.

\section{RESULTADOS}

A continuación, se analizarán las encuestas realizadas a las personas previamente seleccionadas correspondientes a las diferentes comunidades estudiantiles, misma que fue aplicada de manera directa a los interesados. Se presentan las gráficas más representativas de la investigación.

\section{Indique género y edad.}

Después de realizar la prueba piloto,

Figura 1. Género de los encuestados.

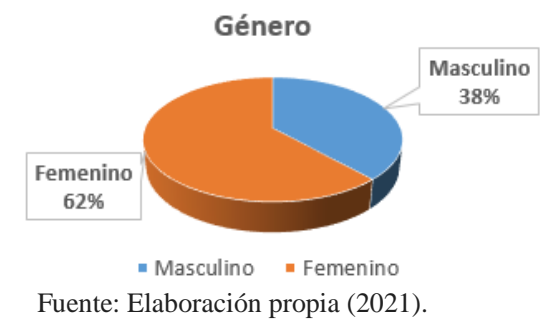

2. Edad de los encuestados.

Figura 2. Edad de los encuestados.

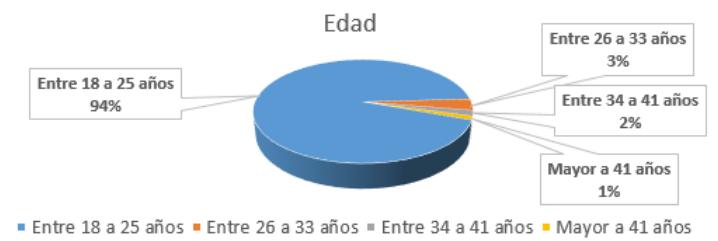

Fuente: Elaboración propia (2021).

Inicialmente se puede observar que el $62 \%$ de los encuestados son mujeres y el $38 \%$ son hombres (Figura 1); en la figura 2, se aprecia que el $94 \%$ tienen entre 18 a 25 años, un $3 \%$ tienen entre 26 a 33 años, el $2 \%$ tienen entre 34 a 41 años y el 1\% tienen más de 41 años. 
Se observa que la mayoría de los encuestados son jóvenes de entre 18 a 25 años, que forman parte de las personas que más se encuentran relacionados con la tecnología.
3. De manera cotidiana, ¿Qué método de pago utiliza más?

Figura 3. Método de pago más utilizado.

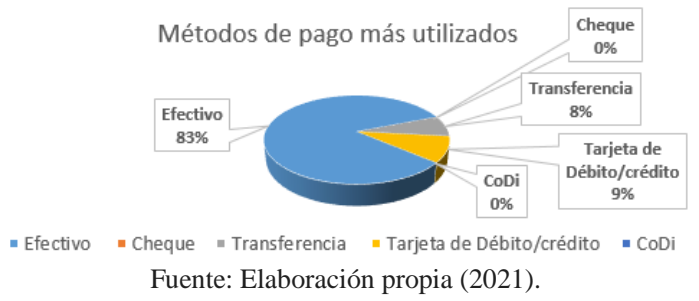

La gran parte de los encuestados utilizan con mayor frecuencia el efectivo en un $83 \%$, en un $9 \%$ las tarjetas de débito/crédito, con un $8 \%$ utilizan transferencias, y ninguno utiliza como método de pago los cheques y el CoDi (Figura 3).

Figura 4. La tecnología implementada en las transacciones económicas.

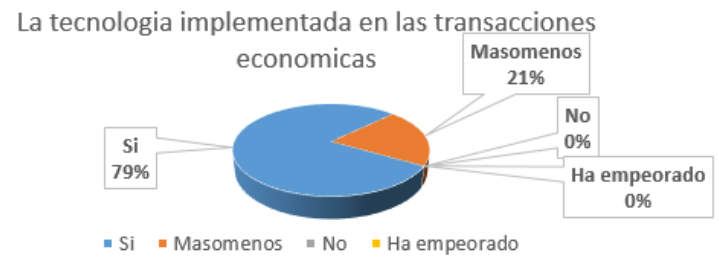

Fuente: Elaboración propia (2021).

diversa cantidad de problemas que estos

Como se puede apreciar en la Figura 4, la tecnología ha beneficiado en mayor proporción a las transacciones económicas con un $79 \%$, mientras que el $21 \%$ restante no están totalmente de acuerdo en las mejoras que se han implementado, por la

Figura 5. Ventajas de la innovación tecnológica en los bancos.

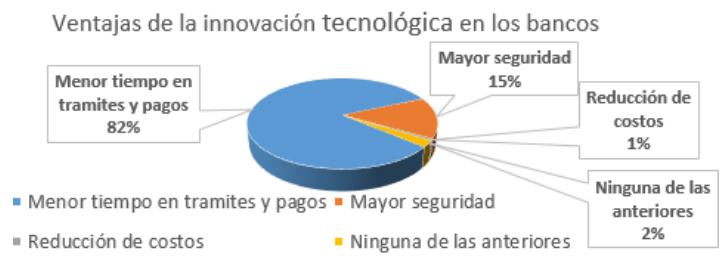

Fuente: Elaboración propia (2021).

Podemos apreciar, que la mayor parte de los encuestado afirmo con un $82 \%$ que el menor tiempo en trámites y pago ha sido la mayor ventaja que trajo consigo la innovación tecnológica en los bancos, así mismo el 15\% mencionan que aumento la seguridad, el $1 \%$ la reducción de costos, y el $2 \%$ ninguna de las opciones anteriores (Figura 5).

6. ¿La innovación tecnología en los bancos ha ocasionado mayor cantidad 
de problemas en los usuarios?

Figura 6. Mayor cantidad de problemas en los usuarios de los bancos.

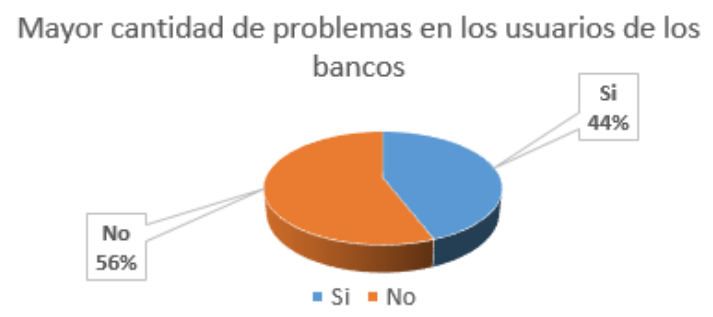

Fuente: Elaboración propia (2021).

El 56\% de los encuestados niega que la innovación tecnológica en los bancos ha ocasionado mayor cantidad de problemas en los usuarios, y 44\% respondieron que sí han aumentado los problemas (Figura 6).

Figura 7. Confianza en los actuales métodos de pago electrónico.

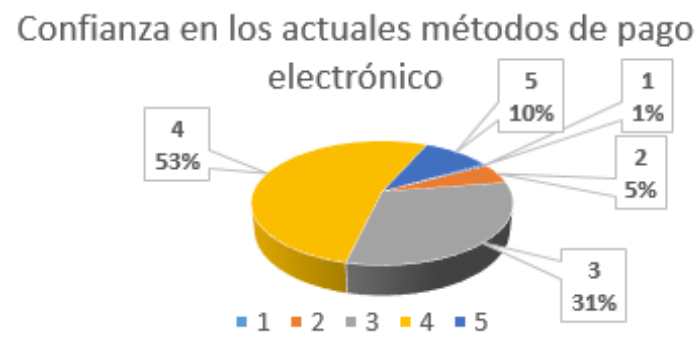

Fuente: Elaboración propia (2021).

El 10\% de los encuestados afirma que, si son muy confiables los actuales métodos de pago electrónico, el 53\% menciona que son confiables, el $31 \%$ afirma una confiabilidad intermedia, el $5 \%$ responde ser poco confiable y el $1 \%$ definitivamente respondió

Figura 8. Conocimiento del entorno de las criptomonedas.

\section{Conocen las criptomonedas}

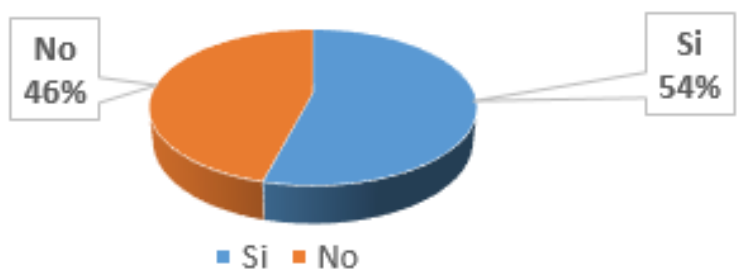

Fuente: Elaboración propia (2021).

digitales toman como base la tecnología

Los proyectos en desarrollo de monedas empleada en las criptomonedas, por lo que es importante, el conocimiento de estas, 
observando que el $54 \%$ de los encuestados si conocen acerca de las criptomonedas, mientras que el $54 \%$ restante las desconocen.

9. ¿Crees posible que en algún momento se deje de utilizar el dinero físico? (Figura 8).

Figura 9. Posibilidad de que en algún momento se deje de utilizar el dinero físico.

\section{Posibilidad de que en algún momento se deje de utilizar el dinero físico}

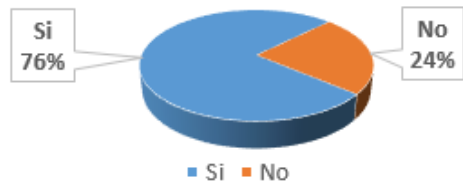

Fuente: Elaboración propia (2021).

y $\tan$ solo el $24 \%$ opinaron lo contrario.

Al paso del tiempo, la tecnología va (Figura 9).

evolucionando en gran medida la forma en que se realizan nuestras actividades cotidianas, por lo que es importante pensar a futuro, y por ello se estableció esta pregunta, donde el $76 \%$ de los encuestados afirman que existe la posibilidad de que en algún

10. Del 1 al 5 ¿Qué tan seguro te sentirías solamente pagando con métodos de pago electrónicos? (Donde 1 es nada seguro y 5 muy seguro) momento se deje de utilizar el dinero físico,

Figura 10. Seguridad de los usuarios al solamente pagar con métodos de pago electrónicos.

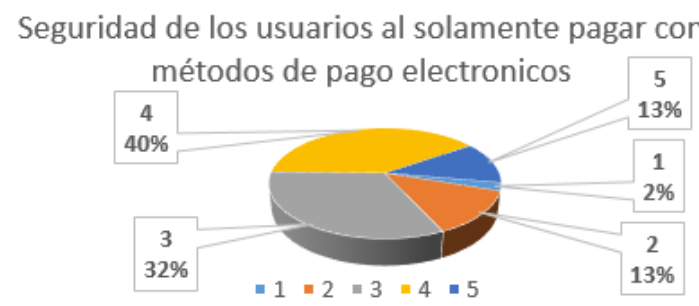

Fuente: Elaboración propia (2021).

Como se puede observar, el $13 \%$ de los encuestados se sentirían muy seguros al solamente poder utilizar métodos de pago electrónico, mientras que el $40 \%$ afirman que es seguro, con un $32 \%$ sentirían tanto seguridad como inseguridad parcial, el $13 \%$ poco seguros y el $2 \%$ restante no se sentirían seguros utilizando únicamente estos medios. (Figura 10).
11. Sí el Banco de México tomara la decisión de desarrollar una moneda digital respaldada por el Peso Mexicano, que tuviera la finalidad de sustituir paulatinamente todo el dinero físico del país en las actividades económicas. Del 1 al 5 ¿Qué te parecería esta idea? (Donde 1 es mala idea y 5 excelente idea)

Figura 11. Aceptación de la idea de desarrollar una moneda digital con respaldo del peso mexicano. 
Aceptación de la idea de desarrollar una moneda

digital con respaldo del peso mexicano

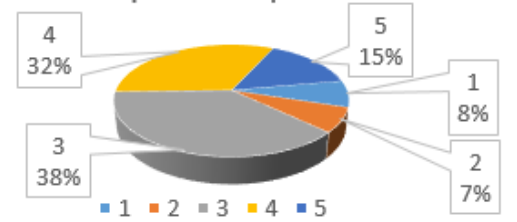

Fuente: Elaboración propia (2021).

Como parte del objetivo de esta investigación, es importante la formulación de esta pregunta, puesto que establece cual sería la aceptación que tendría la idea de desarrollar una moneda digital que cuente con el respaldo del peso mexicano y fuera emitido por el Banco de México, por lo que, de esta manera, podemos apreciar que el $15 \%$ de los encuestados consideran que sería una excelente idea su desarrollo, el $32 \%$

aseguran que sería ideal, el 38\% se encuentra en un punto intermedio, el $7 \%$ considera que la idea es poco aceptable y el $8 \%$ considera que sería una muy mala idea (Figura 11).

12. Con base en el supuesto anterior, ¿Consideras que la sociedad mexicana está preparada para aceptar este cambio en la economía mundial?

Figura 12. Sociedad mexicana preparada para aceptar cambio en la economía mundial.

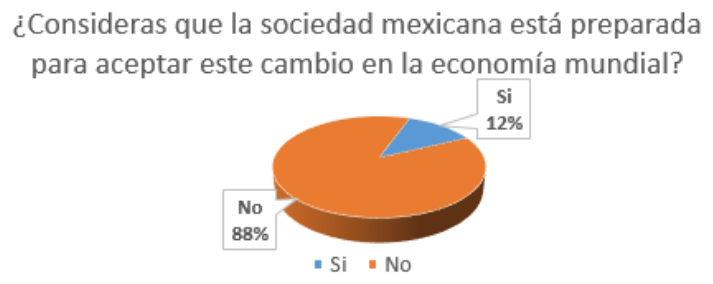

Fuente: Elaboración propia (2021).

Con relación a la pregunta anterior, y que además tiene gran relevancia en este instrumento, se observa que el $88 \%$ los encuestados consideran que la sociedad mexicana no está preparada para sumarse a este cambio de la economía mundial, mientras que solo $12 \%$ considera que si es apta para confrontar este gran cambio. (Figura 12).

\section{CONCLUSIONES}

Con esta investigación se concluye que es necesario comenzar a preparar a la sociedad mexicana para un cambio que se avecina en la economía mundial, donde quizás aún se encuentren en desarrollo los proyectos de monedas digitales soberanas en Asia y Europa, pero en el caso del Yuan Digital ya han comenzado a realizar las pruebas correspondientes para su utilización, por lo que de concretarse este nuevo modelo de monedas digitales, paulatinamente se dejaría de utilizar el dinero físico, lo cual marcaria la pauta en diversos países y en general para el mundo, para comenzar a desarrollar su propias monedas virtuales, y se encuentren a la par de las potencias económicas.

Dicho lo anterior, la investigación nos dejó como resultado, que a pesar de que los jóvenes son las personas que se encuentran mayormente relacionados con la utilización de la tecnología, son los que menor utilizan los métodos electrónicos de pago, ya que durante la vida rutinaria de un mexicano el principal método de pago y el más utilizados es el dinero físico. Por otra parte, las instituciones financieras se han venido a beneficiar gracias a los diversos cambios tecnológicos en el ecosistema, permitiendo mejorar la calidad de las transacciones económicas, sin embargo, como en cualquier contexto no todo es perfecto, puesto que en algunos de los 
casos se ha vuelto un tanto más complejo en su utilización por lo que termina por ocasionar problemas y disgustos en los usuarios. Sin embargo, una de las mayores ventajas que trajo consigo la innovación tecnológica en las instituciones financieras ha sido la reducción del tiempo en los tramites y pagos, ya que por medio de las aplicaciones móviles los usuarios ya no necesitan trasladarse hasta una institución para realizar alguna de estas operaciones, sino que ahora basta con descargar la aplicación del banco móvil, y desde el lugar donde te encuentres puedes realizar los pagos y tramites que necesites. Así mismo, las personas confían en la mayoría de los casos en los actuales métodos de pago digital, sin embargo, como cualquier método existe el riesgo de contraer algún problema.

Con el paso de los años, vemos como nuestro panorama, nuestra vida y sus actividades van cambiando, a la par muchas de las veces de la tecnología, por lo que es imposible no llegar a pensar sobre que en algún momento dejara de utilizarse el dinero físico en todo el mundo, y esa ideología no se encuentran tan lejana de la realidad, puesto que la mayor parte de la personas están conscientes y saben que en algún momento puede pasar, son pocas las personas que no creen posible llegar a no usar más el dinero físico.

Así mismo, solamente un segmento de la población se sentiría seguro al solamente utilizar los métodos de pago electrónicos, mientras que para la otra parte de la sociedad confiaría en ellos, pero aun así sentirían inseguridad sobre su utilización, ya que no todas las personas aceptan en su totalidad el utilizar tecnología en su vida cotidiana, e inclusive muchos de ellos no pueden acceder a este tipo de innovaciones de forma tan sencilla.

Por otra parte, algo muy importante es que la sociedad pese a este tipo de circunstancias, tendría una buena aceptación sobre la idea de desarrollar una moneda digital que fuera respaldada por el peso mexicana y esta fuera emitida por el Banco de México, la cual tendría el objetivo de ir sustituyendo al dinero físico paulatinamente, de tal forma que sería una buena propuesta a futuro para mejorar la eficiencia y el control de las operaciones, sin embargo, el poder desarrollar este tipo de proyectos implicarían incertidumbre sobre cierto segmento de la población, ya que no todas cuentan con las posibilidades para poder sumarse a este tipo de cambios, por lo que la sociedad mexicana realmente no se encuentra preparada para asumir un cambio de esta magnitud, puesto que implica educar y concientizar a la mayor parte de la sociedad sobre como este nuevo modelo de moneda digital podría cambiar el rumbo de un país en cuenta a control, trasparencia y eficiencia en las operaciones.

Respecto a los objetivos planteados, se concluye que con la presente investigación se llegó a conocer y estudiar el uso de los diferentes métodos de pago digital en México, así como la inclusión que tiene la sociedad mexicana hacia estos métodos, para de esta forma determinar su panorama y establecer el posible escenario positivo o negativo que tendría la creación del peso digital respaldado por el Banco de México, afirmando la hipótesis planteado como falsa, debido a los resultados obtenidos se concluye que la creación del peso digital respaldado y emitido por el Banco de México, tiene un escenario incierto, a pesar de que existe una aceptación por parte de la sociedad para utilizarla como método de pago principal, la sociedad mexicana no se encuentra preparada para hacerle frente a este tipo de cambios.

\section{Limitaciones.}

El futuro de las monedas digitales aún es incierto, ya que de momento solamente el Yuan digital se encuentra en periodo de 
pruebas en relación directa con la sociedad del continente asiático. El estudio se limita a estudiar y conocer el panorama económico que presenta la sociedad mexicana para así analizar el escenario que tendría un posible desarrollo de moneda digital respaldado por el peso mexicano y emitido por el Banco de México.

\section{Recomendaciones de carácter académico y prácticos.}

La economía a nivel mundial se encuentra en constante cambio, por lo que es importante el estar siempre al tanto de las nuevas noticias, sobre las nuevas innovaciones en tecnología, así como también conocer sobre las novedades nacientes en cada país, puesto que esto marcara la pauta sobre la preparación de las personas hacia un futuro donde la tecnología se utilizado con mayor frecuencia, al grado de que como mucho expertos mencionan en un futuro no muy lejano se dejara de utilizar el dinero físico, y se tendrán que desarrollar nuevos métodos de pago entre usuarios. 


\section{REFERENCIAS}

Banxico. (mayo de 2018). Banco de México, https://www.banxico.org.mx/spei/d/\%7B44351472054C-58EB-611D-153B1029C2A8\%7D.pdf

BBVA. (2021). BBVA, https://www.bbva.com/es/que-diferencias-hay-entre-una-moneda-digital-yuna-criptomoneda/

Bitcoin México. (2020). Bitcoin México, https://www.bitcoin.com.mx/mexico-y-la-adopcion-decriptomonedas-como-metodo-de-pago/

CNBV. (septiembre de 2020). Comisión Nacional Bancaria y de Valores, https://www.gob.mx/cms/uploads/attachment/file/581089/Panorama_IF_2020.pdf

CNBV. (2020). Comisión Nacional Bancaria y de Valores, https://www.gob.mx/cnbv/prensa/642020-panorama-anual-de-inclusion-financiera-2020?idiom=es

CONDUSEF. (08 de febrero de 2017). CONDUSEF, https://revista.condusef.gob.mx/wpcontent/uploads/2017/02/PDF-s_2017_203_buro.pdf

CONDUSEF. (2020). Comisión Nacional para la Protección y Defensa de los Usuarios de Servicios Financieros, https://www.condusef.gob.mx/?p=estadisticas

Coutiño, R. (2020). ¿Cómo funcionan las transferencias? En Money Coach (págs. 116-117). Ciudad de México: Grupo Empresarial Investormex.

Forbes. (2020). Forbes, https://www.forbes.com.mx/mundo-china-lidera-la-carrera-mundial-por-lamoneda-digital-con-el-yuan-electronico/

García Garza, M. G., Lanzagorta Sánchez, A., \& Ponce González, M. (diciembre de 2020). Vinculategica,

http://www.web.facpya.uanl.mx/Vinculategica/Vinculategica6_2/56_Garcia_Lanzagorta_Pon ce.pdf

Gómez Torres, R. (2021). Cripto Noticias, https://www.criptonoticias.com/regulacion/mexicopodria-verse-obligado-desarrollar-peso-digital-sugiere-

analista/\#: :text=Una\%20versi\%C3\%B3n\%20digital\%20del\%20peso,cadenas\%20de\%20blo ques $\% 20 \mathrm{o} \% 20 \mathrm{blockchain}$.

Gutierrez, F. (2019). El economista, https://www.eleconomista.com.mx/sectorfinanciero/EnMexico-siete-de-cada-10-usuarios-digitales-utilizan-tecnologia-financiera-EY-201906240096.html

Gutierrez, F. (2020). El Economista, https://www.eleconomista.com.mx/economia/Mexico-es-elsexto-pais-con-mayor-adopcion-de-criptomonedas-20200708-0102.html

INEGI. $(23$ de noviembre de 2018). INEGI, https://www.inegi.org.mx/contenidos/saladeprensa/boletines/2018/OtrTemEcon/ENIF2018.p df

LEY FINTECH. (8 de marzo de 2018). Cámara de Diputados, http://www.diputados.gob.mx/LeyesBiblio/pdf/LRITF_090318.pdf

Santander. (2019). Santander, https://www.santander.com.mx/personas/santander-digital/codi.html Visión Industrial. (2020). Visión Industrial, https://www.visionindustrial.com.mx/industria/economia-y-finanzas/el-papel-de-lascriptomonedas-en-la-economia-mexicana 